\title{
May Measurement Month 2019 (MMM19) breaks the ONE MILLION screening milestone for the third year in a row
}

In May 2019, thousands of volunteers from across the globe came together for the third year running to carry out our annual synchronised and standardised blood pressure screening campaign. Since the end of July this year, May Measurement Month (MMM) volunteer statisticians have been tackling the long task of cleaning and analysing the data collected during MMM19, with a view to submitting the results by the end of the year for publication in a leading global journal. Although final numbers have not been confirmed at the time of going to press we are pleased to announce that the number of countries taking part in MMM19 is similar to the number that took part in 2018. Once again those involved have comfortably exceeded targets, by collectively screening in the region of $\mathbf{1 . 5}$
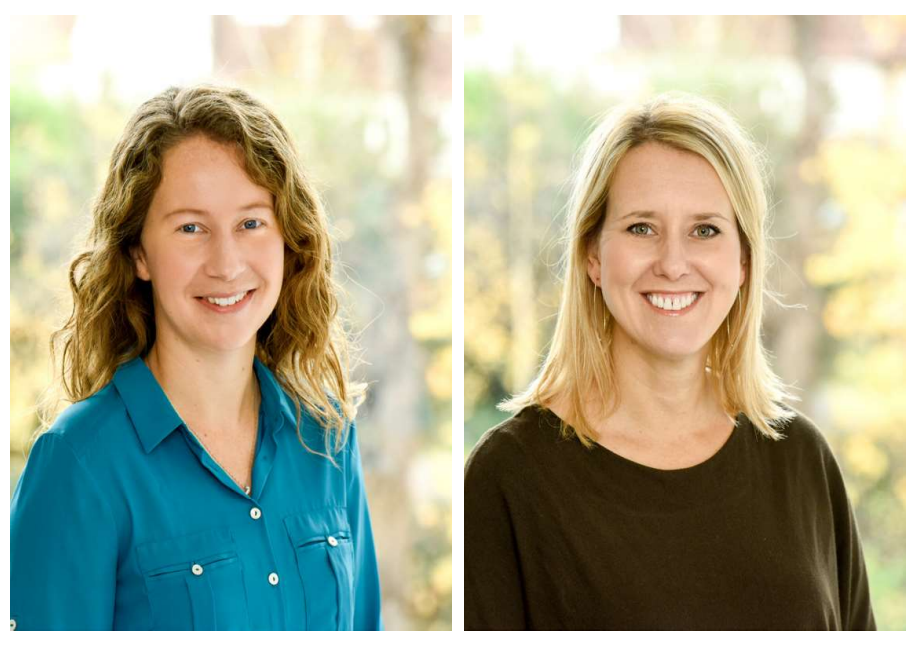
million people.

This continued momentum shows the strong global appetite for, and commitment to, increasing awareness of the issues surrounding high blood pressure via MMM.

\section{New Data in MMM19}

MMM19 saw a few changes compared with previous years. Firstly, we were delighted to welcome a number of new countries who joined MMM for the first time, including Afghanistan, Algeria, Bulgaria, Japan, Kyrgyzstan, Mongolia, Saint Lucia, South Korea, Sri Lanka and Tunisia.

Following feedback from previous MMM campaigns, a number of amendments were made to this year's questionnaire. Four new questions were added to the questionnaire as follows. For those participants who reported taking prescribed medication to treat high blood pressure; we added:

1. How many drug classes do you take for your blood pressure?

2. Do you take a statin?

3. Do you take aspirin?

We also asked:

4. If female, have you had raised blood pressure in a previous pregnancy?

Question 1 will help us to see how frequently monotherapy is successful at controlling BP, whilst questions 2 and 3 will advise on how closely good practice is followed regarding other frequently recommended concomitant medication.

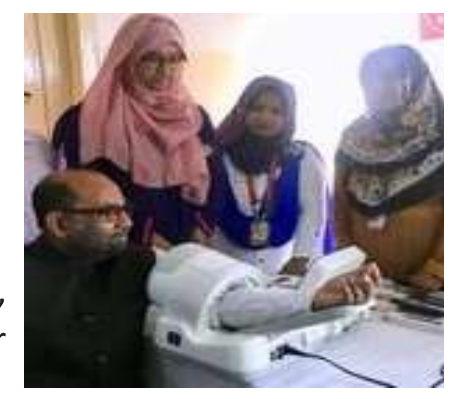

Question 4 will confirm or refute the data suggesting that previous pregnancy associated hypertension is linked with higher BP in later years.

To ensure that the questionnaire was not lengthened, the following questions were removed to make room for the new questions:

1. Time of measurement

2. Temperature at the site of screening

3. What type of BP machine is being used to take the readings? (automated / not automated)

4. Which arm is being used to take the blood pressure? (left / right)

\section{Publications}

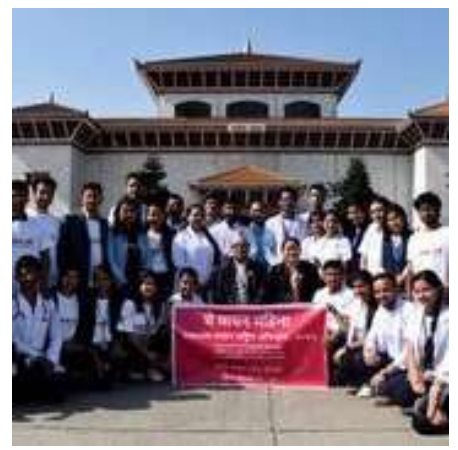

Following the MMM publications in the run up to MMM19**, plans are in the pipeline for a further supplement of national papers from MMM18, as well as the MMM19 global paper both to appear before May 2020. For some analyses we may also combine the data from MMM17, '18 and '19, to provide insights from over 4 million screenees. 
The accuracy of MMM data submitted via the MMM app and Excel spreadsheets has significantly improved over the last 3 years. Whilst the MMM app remains the fastest way for us to collect clean data, at the time of going to press, indications show that whilst the percentage of data submitted via the MMM app has increased it was still disappointingly low at around $15 \%$ or $16 \%$ of data in 2019 compared to $8 \%$ in 2017. Increased app usage in future years would facilitate earlier analysis and therefore potentially allow earlier publication.

Once again, the MMM team would like to thank everyone who has been involved. That MMM helps to save and improve lives was strongly supported by a recent BMJ article (BMJ 2019; 366: L4064) which showed community-based screening in China

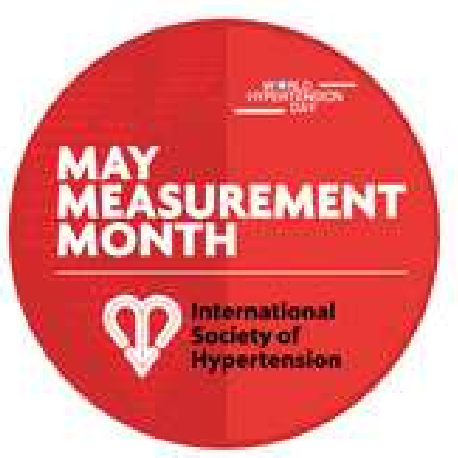
carried out in a very similar fashion to MMM was associated with a large significant benefit on systolic BP levels. However, the MMM campaign cannot be carried out without the dedication and support of the thousands of volunteers who contribute all over the world and without whom there would be no MMM.

If you would like to learn more about future plans for MMM, or would like to get involved in 2020, please contact the MMM Project Manager: manager@maymeasure.com

A Simple Measure to Save Lives

Be part of it!

www.maymeasure.com

NOTE:

May Measurement Month is an initiative of the International Society of Hypertension launched in 2017 to build upon and extend WHL's World Hypertension Day.

** Published papers in 2019:
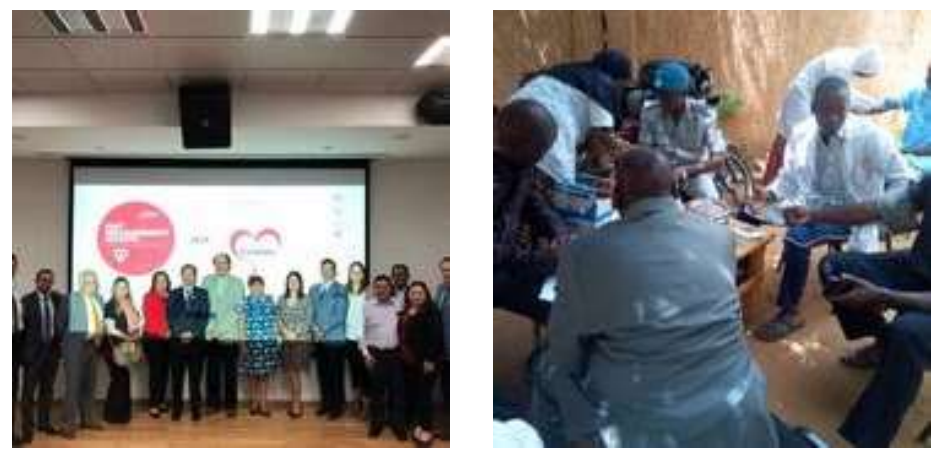

- MMM17 39 national papers supplement https://academic.oup.com/eurheartj/article/40/25/2006/5481538

- MMM18 global analysis https://academic.oup.com/eurheartj/article/40/25/2006/5481538

\section{"DDD": DYLAN'S DISTRIBUTION DATA}

The July 2019 issue of Hypertension News maintained strong readership, albeit somewhat lower than our peak readership with the March 2019 issue. Part of this may be attributed to summer holiday which inevitably leads to reduced readership and engagement.

\section{Dylan's Distribution Data (July-September 2019)}

\begin{tabular}{l}
\hline Total Estimated Readership \\
\hline Accessed via Twitter \\
\hline Accessed via Facebook \\
\hline Accessed via DOI \\
\hline Accessed via Web Site
\end{tabular}

\section{4 \\ 184 \\ 155 \\ 2611 \\ 574}

